\title{
Use of family member-based supervision in the management of patients with hypertension in rural China
}

\author{
Susan Huang ${ }^{1,2, *}$ \\ Ye Chen ${ }^{2, *}$ \\ jing Zhou ${ }^{2}$ \\ Jianming Wang ${ }^{1,2}$ \\ 'Department of Social Medicine \\ and Health Education, ${ }^{2}$ Department \\ of Epidemiology and Biostatistics, \\ School of Public Health, Nanjing \\ Medical University, Nanjing, People's \\ Republic of China \\ *These authors contributed equally \\ to this work
}

This article was published in the following Dove Press journal:

Patient Preference and Adherence

31 July 2014

Number of times this article has been viewed
Abstract: Medication nonadherence is a major problem in the management of hypertension. The aim of this study was to develop a family member-based supportive therapy for patients with hypertension to provide an affordable way to access essential health services and to ensure adequate control of blood pressure. This study applied a mixed methods approach using qualitative and quantitative study designs in Yangzhong County, a rural area in the People's Republic of China. Findings from indepth interviews demonstrated that the limited effects of traditional health education, a lack of professional advice regarding antihypertensive treatment, and age were related to a patient's adherence with regular blood pressure measurement and taking medication. We also performed a quantitative study, selecting two villages in Yangzhong County as study sites. A total of 188 patients with hypertension were invited to participate in a 6-month family member-based intervention trial. The primary outcomes were the acceptability and feasibility of the intervention strategy. Secondary outcomes included medication adherence and changes in blood pressure. More than $75 \%$ of patients expressed a wish for external reminders, and $93.5 \%$ responded that they would accept the family member-based supervision. The patients preferred their spouse or a child as the supervisor. After the 6-month intervention, the proportion of patients with uncontrolled blood pressure decreased from $87.2 \%$ to $45.7 \%$. This pilot study shows that external supervision by family members is acceptable and feasible for patients with hypertension; it also shows favorable effects with regard to improved treatment adherence and blood pressure control. Future randomized controlled trials with modified intervention measures are needed to validate this finding.

Keywords: hypertension, treatment, adherence, supervision, intervention

\section{Background}

Despite the progress made in the prevention and treatment of high blood pressure (BP), hypertension remains a major public health challenge. ${ }^{1-3}$ High BP increases the risk of mortality and morbidity from stroke, coronary heart disease, congestive heart failure, and end-stage renal disease, and negatively impacts quality of life. ${ }^{1}$ However, BP remains inadequately controlled in most individuals with hypertension. If BP control could be improved, cardiovascular morbidity and mortality would be decreased significantly. ${ }^{4}$

Nonadherence with medication is a major problem in the management of hypertension. ${ }^{5}$ Improving patient adherence with antihypertensive medication is an important strategy for BP control. ${ }^{6}$ Previous experience in the management of tuberculosis might provide a good model for managing hypertension. Tuberculosis and hypertension have a lot of similarities: both are commonly occurring chronic 
diseases and remain major public health issues in the People's Republic of China; both need therapies consisting of a cocktail of drugs taken over a long period; adverse drug reactions and nonadherence present major barriers for effective control; and family and social support are necessary for treatment and prevention of the disease. DOTS (direct observed therapy, short course) remains at the heart of the Stop TB Strategy recommended by the World Health Organization. ${ }^{7}$ One key component of DOTS is supervised treatment, which could be more simply described as directly observed therapy (DOT). The aim of DOT is to help patients to take drugs regularly, ensure adherence to a long-term treatment to achieve a satisfactory cure rate, and prevent potential drug resistance. ${ }^{7}$ DOT is regarded as a main component of the "breakthrough" in the tuberculosis control program that has been widely promoted globally. ${ }^{8}$

The success of DOT in patients with tuberculosis provides us with clues for setting up a similar essential service package for patients with hypertension. ${ }^{9}$ However, DOT supervised by clinical doctors is not feasible in patients with hypertension because antihypertensive treatment is lifelong, whereas traditional antituberculosis therapy only lasts 6-8 months. We hypothesized that patients can be supervised by laypersons, such as family members, instead of doctors, to achieve satisfactory treatment adherence and control of BP.

We performed this pilot study to evaluate the acceptability and feasibility of the family member-based supervision program for patients with essential hypertension, with the aim of providing them with an affordable way to access essential health care services and to ensure adequate control of BP.

\section{Materials and methods}

\section{Ethical considerations and clinical trial registration}

The study was approved by the institutional review board of Nanjing Medical University. Written informed consent was obtained from all participants. We registered this study on the Chinese Clinical Trials Register (http://www.chictr.org/cn/; registration number ChiCTR-ONC-12001940).

\section{Study design and data collection}

This study applied a mixed methods approach using a qualitative and quantitative design. We purposely selected Yangzhong County as the study area. Yangzhong is an island located in the middle of the Yangtze River in the southeastern part of Jiangsu Province, People's Republic of China, and has a population of 0.3 million and an area of $332 \mathrm{~km}^{2}$. We randomly selected two villages from Baqiao Town in Yangzhong County as the study sites. Using the local disease surveillance system, we recruited 210 patients with hypertension. The inclusion criteria were: essential hypertension; willingness to participate in this study; absence of hypertension-related complications; and residence with family members. After preliminary examination, we excluded 22 patients who did not meet the inclusion criteria $(n=8)$, declined to participate $(n=10)$, or could not be interviewed for other reasons $(n=4)$. Finally, 188 patients with essential hypertension were recruited as the study subjects in February 2012. Face-to-face interviews were performed in the village conference room using a structured questionnaire. We recorded each patient's basic demographic characteristics, socioeconomic status, treatment history, adherence with antihypertensive medication, and attitudes toward family member-based supervision. The patient's BP was measured in $\mathrm{mmHg}$ using a mercury sphygmomanometer and was recorded in the form of two numbers, ie, systolic BP and diastolic BP. After the baseline investigation, we performed a 6-month self-controlled intervention trial. The intervention included the following elements: patients selected a family member as the observer to supervise their treatment; family observers were trained regularly; family observers supervised the patients taking their drugs and recorded their adherence; and observers were responsible for reminding patients to seek timely health care and to get their BP monitored on a regular basis. Face-to-face interviews were performed again at the end of the pilot study stage (September 2012) to collect outcomes, including medication adherence, changes in systolic and diastolic BP, and the percentage of patients with adequately controlled BP. A flowchart of the trial is shown in Figure 1.

We also performed a qualitative study by inviting 16 patients (six from Sanwei village and ten from Leyuan village) and two village doctors for indepth interviews. A semischematized guide covering both general and specific questions was used for collection of qualitative data. The indepth interviews focused on the patients' health care seeking behavior, knowledge about hypertension, medication adherence, and attitudes toward the proposed health care services for hypertension. The themes used to interview the village doctors included the current hypertension control program and treatment strategy, treatment adherence of patients in the local villages, the main reasons for nonadherence, problems faced by local health care workers, and their suggestions for the current program 


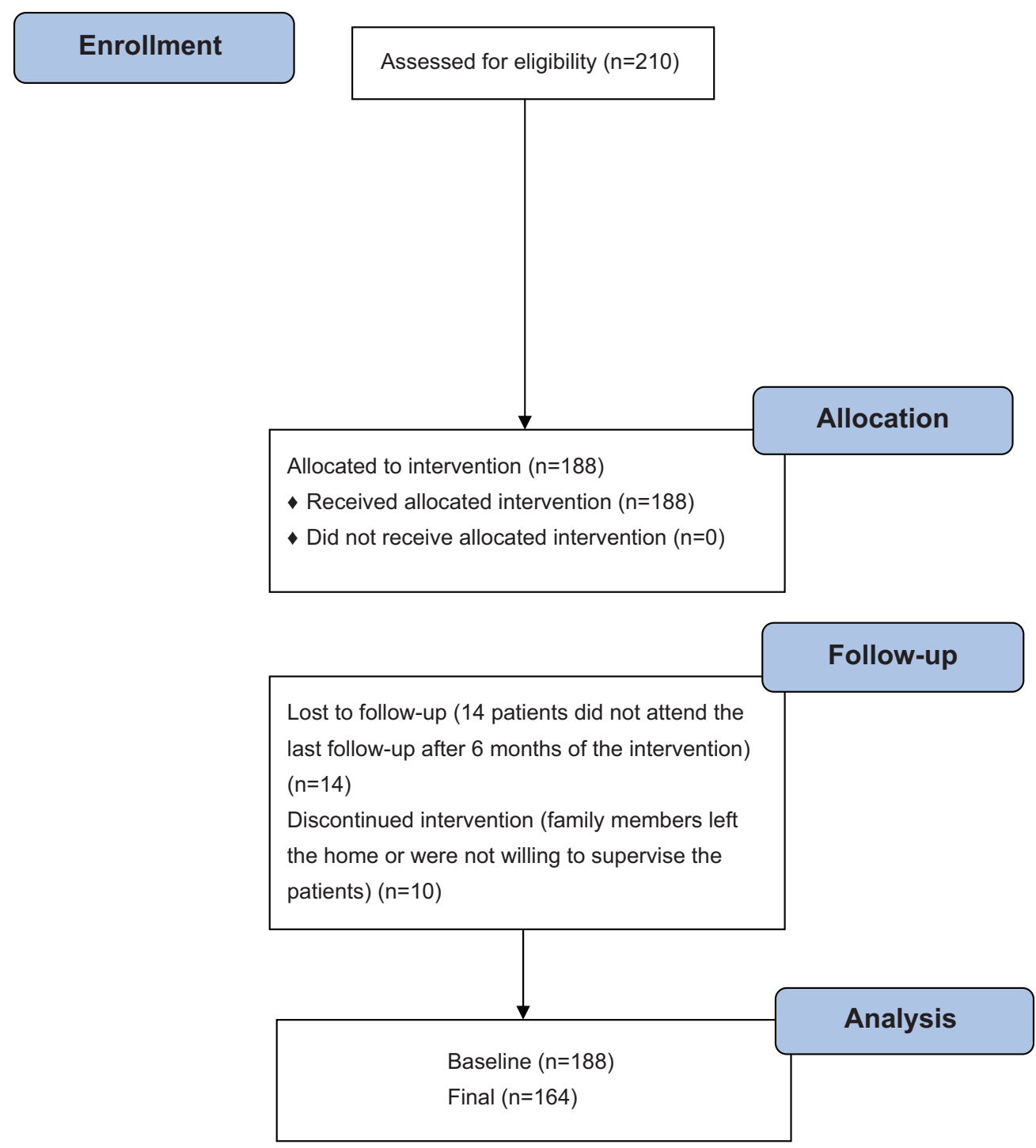

Figure I Flow chart of the trial.

of managing hypertension. The interviews were conducted at local health facilities in the Chinese Mandarin language, lasted for 20-40 minutes, and were tape-recorded with each respondent's permission.

\section{Data analysis}

For the quantitative study, the data were entered using EpiData software (Odense, Denmark) and analyzed using Stata version 10.0 (Statcorp LP, College Station, TX, USA). For the continuous outcomes, such as BP, a Student's $t$-test was used to compare the effect of the intervention. For binary outcomes, a chi-squared test was used to estimate the effect of the intervention. Primary outcomes included the acceptability and feasibility of this family member-based supervision, and secondary outcomes included medication adherence, changes in $\mathrm{BP}$, and $\mathrm{BP}$ control rate.
Data from the indepth interviews were analyzed to "offer insight, enhance understanding, and provide a meaningful guide to action". As recommended for qualitative studies, the data analysis and collection proceeded simultaneously. In addition to the tape recordings, the interviewers kept field notes documenting their immediate perceptions of the session. The recorded interviews were transcribed verbatim and later compared with the audio records to ensure the accuracy of the transcripts. The transcripts and notes were analyzed thematically by creating coding nodes for common themes and subthemes. The codes were developed based on the original terms used by the participants. Tentative categories and subcategories were created, and several major themes emerged from the clustered codes based on the patterns and relationships between the categories. The categories were considered saturated when no new themes appeared. 
The patients were asked to compare the themes developed by the researchers with their own perspectives and experiences, and this ultimately ensured an accurate representation of the participants' perspectives. Additional validation was obtained by discussions with local public health workers regarding our findings. All quotes from participants are indicated in italics.

\section{Results}

\section{Findings from the self-controlled} intervention trial

\section{Basic characteristics of study subjects}

In total, we recruited 188 patients with essential hypertension from two villages in Yangzhong County. As shown in Table 1, 64 (34.0\%) were men and 124 (66.0\%) were women. The average patient age was $64.4 \pm 10.7$ years, $42.6 \%$ were illiterate, and $88.8 \%$ were married (Table 1 ).

\section{Knowledge and attitudes toward antihypertensive treatment at baseline}

Thirty-three percent of the patients were not aware of the normal range of $\mathrm{BP}$, and $31.4 \%$ measured their $\mathrm{BP}$ less than once per month. Regardless of the severity of their hypertension, $6.4 \%$ did not take any antihypertensive drugs. Only $64.4 \%$ of patients reported adequate adherence with antihypertensive treatment (Table 2).

\section{Effects of family member-based supervision}

At baseline, $82.4 \%$ of patients had a systolic BP $>140 \mathrm{mmHg}$ and $60.6 \%$ had a diastolic $\mathrm{BP}>90 \mathrm{mmHg}$. In total, $87.2 \%$ had uncontrolled BP. After intervention, the proportion of patients with uncontrolled BP decreased to $45.7 \%$. As shown in Table 3, there were significant changes in BP values and

Table I Basic characteristics of the study subjects

\begin{tabular}{lll}
\hline Characteristics & $\mathbf{n}$ & $\%$ \\
\hline Sex & & \\
$\quad$ Men & 64 & 34.0 \\
$\quad$ Women & 124 & 66.0 \\
Village & & \\
$\quad$ Leyuan & 90 & 47.9 \\
$\quad$ Sanwei & 98 & 52.1 \\
Education & & \\
$\quad$ Illiterate & 80 & 42.6 \\
$\quad$ Primary school & 64 & 34.0 \\
$\quad$ Middle school & 27 & 14.4 \\
$\quad$ Senior high school or higher & 17 & 9.0 \\
Marital status & & \\
$\quad$ Married & 167 & 88.8 \\
Single, divorced, or widowed & 21 & 11.2 \\
\hline
\end{tabular}

Table 2 Knowledge of hypertension and attitudes to antihypertensive treatment at baseline

\begin{tabular}{lll}
\hline Questions & $\mathbf{n}$ & $\%$ \\
\hline Know the normal range of blood pressure & & \\
No & 62 & 33.0 \\
Yes & 126 & 67.0 \\
Frequency of blood pressure measurement & & \\
At least once weekly & 38 & 20.2 \\
Once a month & 91 & 48.4 \\
Once every 3 months & 18 & 9.6 \\
Once every 6 months & 11 & 5.9 \\
Less than once every 6 months & 24 & 12.8 \\
Irregular & 6 & 3.2 \\
Taking antihypertensive drugs & & \\
No & 12 & 6.4 \\
Yes & 176 & 93.6 \\
Adherence to treatment (self-reported) & & \\
No & 67 & 35.6 \\
Yes & 121 & 64.4 \\
\hline
\end{tabular}

the controlled BP rate after intervention. The paired chisquared test also showed a significant increase in the BP control rate before and after intervention $(P<0.001)$.

\section{Acceptability of family member-based supervision}

At baseline, $75.7 \%$ of patients reported that they needed external supervision for BP measurement and antihypertensive treatment, and $93.5 \%$ indicated that they would like to be supervised by family members. The patients preferred their spouse $(54.4 \%)$ or children $(42.2 \%)$ as supervisors (Table 4). After 6 months of the intervention, $68.9 \%$ of patients were being continuously supervised by family members. Of these, $59.3 \%$ were supervised by their spouse, while $31.9 \%$ were supervised by their children. Of those supervised by family members, $75.2 \%$ regarded the supervision as effective (Table 5). During the intervention stage, we also designed a calendar for family members to record the patient's medication adherence, and $68.2 \%$ kept records on the calendar on a regular basis. On further

Table 3 Blood pressure before and after intervention

\begin{tabular}{llll}
\hline Blood pressure & $\begin{array}{l}\text { Baseline } \\
(\mathbf{n}=\mathbf{1 8 8})\end{array}$ & $\begin{array}{l}\text { After } \\
\text { intervention } \\
(\mathbf{n}=164)\end{array}$ & P-value \\
\hline SBP (mean $\pm \mathrm{SD}, \mathrm{mmHg})$ & $165.3 \pm 22.8$ & $141.2 \pm 17.3$ & $<0.001$ \\
DBP (mean $\pm \mathrm{SD}, \mathrm{mmHg})$ & $96.9 \pm 15.4$ & $83.1 \pm 10.0$ & $<0.001$ \\
Controlled blood pressure* & & & \\
Yes & 24 & 89 & $<0.001$ \\
No & 164 & 75 & \\
\hline
\end{tabular}

Notes: *SBP, < $140 \mathrm{mmHg}$; DBP, $<90 \mathrm{mmHg}$.

Abbreviations: DBP, diastolic blood pressure; SBP, systolic blood pressure; SD, standard deviation. 
Table 4 Attitudes to the family member supervision strategy at baseline

\begin{tabular}{lll}
\hline Questions & $\mathbf{n}^{*}$ & $\%$ \\
\hline $\begin{array}{l}\text { Do you need external supervision for blood } \\
\text { pressure measurement and antihypertensive }\end{array}$ & & \\
treatment? & & \\
$\quad$ No & 45 & 24.3 \\
$\quad$ Yes & 140 & 75.7 \\
Would you like to choose one family & & \\
member to supervise you? & 12 & \\
$\quad$ No & 173 & 6.5 \\
Yes & & 93.5 \\
Which family member do you prefer? & $\mathrm{I}$ & 0.6 \\
$\quad$ Parents & 98 & 54.4 \\
Spouse & 76 & 42.2 \\
Children & 5 & 2.8 \\
Others & & \\
\hline
\end{tabular}

Note: *There were some missing values.

checking, we found that $43.3 \%$ were completely recorded, $29.2 \%$ were partly recorded, $11.7 \%$ were lost, and $15.8 \%$ were not recording any details of treatment adherence or BP values.

\section{Findings from the qualitative study}

Several themes emerged during indepth interviews with the patients and village doctors.

\section{Health education}

The patients acknowledged that health education about common diseases has been strengthened significantly in recent years. They mentioned that the traditional health education programs usually focused on providing materials, such as leaflets, posters, or books. However, many rural patients were illiterate or semiliterate, and had difficulty reading and understanding such materials.

Table 5 Family member-based supervision after 6 months of intervention

\begin{tabular}{lll}
\hline Questions & $\mathbf{n}$ & $\%$ \\
\hline Were you supervised by family members? & & \\
No & $5 \mathrm{I}$ & $3 \mathrm{I} . \mathrm{I}$ \\
Yes & 113 & 68.9 \\
If you were supervised by family & & \\
members, do you think it was effective? & & \\
No & 28 & 24.8 \\
Yes & 85 & 75.2 \\
Who was the main supervisor? & & \\
Parent & 1 & 0.9 \\
Spouse & 67 & 59.3 \\
Child & 36 & 31.9 \\
Other & 9 & 8.0 \\
\hline
\end{tabular}

"We have received a lot of materials. However, we are old and can't read and understand. Only when my children return home, I can give it to them and ask them to explain. They are so busy and seldom have free time to read it." (Female patient, aged 75 years).

Both patients and their health care providers thought that new methods should be developed to serve the general population.

"I have sent out thousands of educational materials to the habitants living in my community. It costs a lot, but people seldom read it. I believe that its effect is limited. It is better to adopt some cost-effective way to educate people, change their attitudes and practices." (Village doctor).

Age

Hypertension does not affect only the elderly population. With changes in lifestyle and behavior, more and more young people are developing hypertension. However, young people often pay less attention to the disease and ignore its adverse outcomes.

"I am a patient with hypertension. My son and daughter were also diagnosed with hypertension several years ago. They are fat, but they all refuse to take antihypertensive drugs. I have no idea. I think they should be warned about the potential risks of uncontrolled blood pressure." (Male patient, aged 74 years).

"I am diagnosed with hypertension. My son also has hypertension. His systolic BP is $150 \mathrm{mmHg}$. This value lasted for a long time; thus, he regards it as a stable blood pressure value. He doesn't want to take antihypertensive drugs. I am worrying about him." (Female patient, aged 60 years).

\section{Professional advice}

The patients usually bought their drugs from drug stores, following the recommendations of clerks, friends, or advertisements on the radio or television. Some elderly patients used drugs that were provided by their children, and seldom sought professional advice for treatment of their hypertension. They preferred to choose or change the brand or dose of their drugs based on their own experience.

"Sometimes my blood pressure is normal. Sometimes it reaches $160 \mathrm{mmHg}$. I don't know why. If the blood pressure is abnormal, I will take more drugs. Now I take four types of drugs per day. My daughter bought these drugs for me. I don't know the brand of these drugs." (Male patient, aged 49 years).

"I have been diagnosed with hypertension for 7 to 8 years. After 3 years' treatment, the blood pressure stayed normal. 
Then I cut down the dose of antihypertensive drugs. Last month, the blood pressure was elevated again, and then I raised the dose of drugs. Now, the blood pressure is $130 / 80 \mathrm{mmHg}$, which is normal again." (Female patient, aged 66 years).

\section{External reminders}

Hypertension is a lifelong disease. The patients acknowledged that external help, such as supervision or reminders, was important. Family members were the best choice for a supervisor, and could ensure accessibility for a large proportion of patients.

"My wife and daughter often remind me to take drugs and urge me to measure the blood pressure regularly. Though the health education is important, external supervision is also necessary for us." (Male patient, aged 49 years).

Some patients also developed specific methods to remind themselves to take their drugs.

"I put drugs on the dining table. Every day, when I prepare to eat, I will see the drugs. It reminds me to take drugs." (Female patient, aged 66 years).

Some patients thought that the calendar we designed could be a useful tool to assist them in increasing their adherence with treatment.

"I put the calendar on the table. My husband marks the day with a ' $\sqrt{ }$ ' when I take the drugs. It reminds me to take drugs every day, and it is clear to me." (Female patient, aged 62 years).

\section{Discussion}

The prevalence of hypertension in the People's Republic of China is similar to that in other countries, but the proportion of people with controlled BP has remained low for the past few decades. ${ }^{10}$ According to the Fourth National Health and Nutrition Survey (2002), the prevalence, treatment, and control rates for hypertension were $18.8 \%, 24.7 \%$, and $6.1 \%$, respectively..$^{10}$ Further research is required to improve our understanding of the pathophysiology of hypertension and its management in the People's Republic of China. This pilot study took a mixed methods approach with a quantitative and qualitative design to explore the effects of family member-based supervision. The findings suggest that such supervision might be an alternative way to remind patients to measure BP and to take drugs regularly.

Despite the availability of more than 75 antihypertensive agents, blood pressure control is still inadequate. ${ }^{2}$ Adverse drug reactions should be identified early and managed promptly. Treatment should be monitored because better adherence with antihypertensive medication can control BP effectively and reduce the risk of developing cardiovascular disease. $^{11}$

Although the newly formed basic public health service program in the People's Republic of China covers a wide range of measures to prevent and manage chronic diseases such as hypertension, standardized antihypertensive therapy and management has not yet been integrated with the disease prevention and case monitoring system. Patients tend to seek health care at different levels of the hospital system based on their knowledge, attitudes, insurance, and financial situation. Few of them are routinely monitored for adverse drug reactions and treatment adherence, resulting in uncontrolled BP and a high risk of developing the complications of hypertension. There is an obvious gap between the services provided by community public health facilities and those provided by hospitals. Traditional medical care focuses on one-on-one doctor-patient interactions at any medical center visit, which misses many opportunities to influence patient care in a positive way. It is estimated that only $20 \%$ of patients' health care takes place in a doctor's office, and that the other $80 \%$ occurs in the home. ${ }^{12}$ Thus, family-based case management is of particular interest because it provides assistance to patients in the management of their chronic conditions. Well planned, proactive, and reactive care improves intermediate and long-term disease outcomes. ${ }^{4}$

In this pilot study, we designed a family member-based supervision package that aimed to provide external assistance to patients in a cost-effective and acceptable way. After 6 months of intervention, we observed a significant improvement in the treatment adherence and BP control rate. Although the limitations of this self-controlled trial are obvious, the findings of this study can at least in part give clues for future research on family-based external supervision of patients with hypertension.

In recent years, an increasing number of studies have been performed to explore innovative ways to improve a patient's treatment adherence and outcomes, such as physician-level and practice-level financial incentives for treatment of hypertension, ${ }^{13,14}$ shared decision-making in antihypertensive therapy, ${ }^{15}$ interdisciplinary education programs,${ }^{16}$ and home-based BP telemonitoring. ${ }^{17}$ Considering that the reasons for noncompliance are complicated, individualized intervention strategies should be designed based on the characteristics of the patient and the local context.

In addition to the preliminary findings regarding the family member-based supervision program for the management of hypertension, we also identified some specific 
characteristics of the health-seeking behavior of patients in the study area. A large proportion of the patients sought services at a drug store or low-level health care facilities, and some patients took antihypertensive drugs without a formal clinical consultation or regular BP monitoring. This information provides clues for modification of intervention strategies in the future. It is planned to invite specialized clinicians to give lectures and on-site consultations for both patients and their family members. This pilot study offers suggestions for a future expanded research study, which should consider further questions, as follows.

\section{How could the effects of external supervision be strengthened?}

In addition to educating family members, we could continue to use a specific calendar to help record BP values and to estimate a patient's treatment adherence. Short text messages can be sent regularly to family members who have mobile phones. Local village doctors or health workers can contact the patients, as well as their family members, through phone calls or home visits.

\section{How should BP be controlled}

\section{if a patient's adherence is good?}

In the expanded study, we will add more interventions to the education package, including inviting specialist doctors to give lectures and provide consultations, recommending those who have good adherence but poor BP control to visit higher-level hospitals for health care, and recommending that hypertensive patients undertake physical exercise and adjust their diet. ${ }^{18}$

\section{Limitations}

There are several limitations to our study. First, it was a pilot study that used a quasi-experimental design (pre-post comparison without a control group), so it may not be possible to convincingly demonstrate a causal link between the intervention and the observed outcomes. Further randomized controlled trials are necessary to validate our findings. Second, 24 patients did not attend the last follow-up visit at the end of the intervention period for a variety of reasons (14 patients were lost to follow-up, and ten patients' family members had left the home or were not willing to supervise). We further compared the basic characteristics, such as age and sex, of the study subjects between those lost to follow-up and those retained in the study, and the difference was not statistically significant. Third, this study was performed in two villages from one rural county so selection bias was unavoidable, which makes it difficult to generalize our findings to other areas without validation studies.

\section{Conclusion}

In this pilot study, external supervision was essential for BP control. Family member-based supervision was acceptable and feasible for patients with hypertension in the rural areas of the People's Republic of China and showed positive effects on treatment adherence and the BP control rate. Future randomized controlled trials with modified intervention measures are needed to validate these findings.

\section{Acknowledgments}

This study is partly supported by grants from the China Medical Board (CMB991), the National Student Innovation Training Program (201210312004), the Jiangsu Student Innovation Training Program (2012JSSPITP1019), and the Priority Academic Program Development of Jiangsu Higher Education Institutions.

\section{Disclosure}

The authors report no conflicts of interest in this work.

\section{References}

1. Israili ZH, Hernandez-Hernandez R, Valasco M. The future of antihypertensive treatment. Am J Ther. 2007;14(2):121-134.

2. Mensah GA, Bakris G. Treatment and control of high blood pressure in adults. Cardiol Clin. 2010;28(4):609-622.

3. Cheung BM. The hypertension-diabetes continuum. J Cardiovasc Pharmacol. 2010;55(4):333-339.

4. Alderman MH. Blood pressure control to reduce cardiovascular morbidity and mortality: today and tomorrow. Eff Clin Pract. 1998;1(1): 23-25.

5. Kretchy I, Owusu-Daaku F, Danquah S. Spiritual and religious beliefs: do they matter in the medication adherence behaviour of hypertensive patients? Biopsychosoc Med. 2013;7(1):15.

6. Lewis LM, Ogedegbe C, Ogedegbe G. Enhancing adherence of antihypertensive regimens in hypertensive African-Americans: current and future prospects. Expert Rev Cardiovasc Ther. 2012;10(11):1375-1380.

7. Xianyi C, Fengzeng Z, Hongjin D, et al. The DOTS strategy in China: results and lessons after 10 years. Bull World Health Organ. 2002; 80(6):430-436.

8. Garner P. What makes DOT work? Directly observed therapy. Lancet. 1998;352(9137):1326-1327.

9. Hu D, Liu X, Chen J, et al. Direct observation and adherence to tuberculosis treatment in Chongqing, China: a descriptive study. Health Policy Plan. 2008;23(1):43-55.

10. Wang JG, Li Y. Characteristics of hypertension in Chinese and their relevance for the choice of antihypertensive drugs. Diabetes Metab Res Rev. 2012;28 Suppl 2:67-72.

11. Corrao G, Parodi A, Nicotra F, et al. Better compliance to antihypertensive medications reduces cardiovascular risk. J Hypertens. 2011;29(3):610-618.

12. Green BB, Ralston JD, Fishman PA, et al. Electronic communications and home blood pressure monitoring (e-BP) study: design, delivery, and evaluation framework. Contemp Clin Trials. 2008;29(3):376-395. 
13. Petersen LA, Simpson K, Pietz K, et al. Effects of individual physician-level and practice-level financial incentives on hypertension care: a randomized trial. JAMA. 2013;310(10):1042-1050.

14. Dolor RJ, Schulman KA. Financial incentives in primary care practice: the struggle to achieve population health goals. JAMA. 2013;310(10):1031-1032.

15. Tinsel I, Buchholz A, Vach W, et al. Shared decision-making in antihypertensive therapy: a cluster randomised controlled trial. $B M C$ Fam Pract. 2013;14(1):135.
16. Lauziere TA, Chevarie N, Poirier M, Utzschneider A, Belanger M. Effects of an interdisciplinary education program on hypertension: a pilot study. Can J Cardiovasc Nurs. 2013;23(2):12-19.

17. Margolis K, Asche S, Bergdall A, et al. A3-1: outcomes of a randomized trial of home blood pressure telemonitoring with pharmacist case management. Clin Med Res. 2013;11(3):134.

18. Zhang J, Xu AQ, Ma JX, et al. Dietary sodium intake: knowledge, attitudes and practices in Shandong Province, China, 2011. PLoS One. 2013;8(3):e58973.

\section{Publish your work in this journal}

Patient Preference and Adherence is an international, peer-reviewed, open access journal that focuses on the growing importance of patient preference and adherence throughout the therapeutic continuum. Patient satisfaction, acceptability, quality of life, compliance, persistence and their role in developing new therapeutic modalities and compounds to optimize clinical outcomes for existing disease states are major areas of interest for the journal. This journal has been accepted for indexing on PubMed Central. The manuscript management system is completely online and includes a very quick and fair peer-review system, which is all easy to use. Visit http://www. dovepress.com/testimonials.php to read real quotes from published authors.

Submit your manuscript here: http://www.dovepress.com/patient-preference-and-adherence-journal 